\title{
Estimation of Conditional Survival Function under Dependent Right Random Censored Data
}

\author{
Abdushukurov Abdurahim Ahmedovich \\ Tashkent Branch of Moscow State University named after M.V. Lomonosov
}

\begin{abstract}
In this paper considered problem consist in estimation of conditional survival function by right random censoring model in the presence of covariate. We propose a new estimator of conditional survival function and study its large sample properties. We present result of asymptotic normality with the same limiting Gaussian process as for copula-graphic estimator.
\end{abstract}

Keywords: survival function, random censoring, covariate, copula function, copula-graphic estimator.

\section{Introduction}

In such applied areas as bio-medicine, engineering, insurance and social sciences researchers are interested in positive variables, which are expressed as a time until a certain event. For example, in medicine the survival time of individual, while in industrial trials, time until break down of a machine are non-negative random variables (r.v.-s) of interest. But in such practical situations, the observed data may be incomplete, that is censored. This is the case, for example, in medicine when the event of interest-death due to a given cause and the censoring event is death due to other cause. In industrial study, it may occur that some piece of equipment is taken away (that is censored) because it shows some sign of future failure. Moreover, the r.v.-s of interest (lifetimes, failure times) and censoring r.v.-s usually can be infuenced by other variable, often called prognostic factor or covariate. In medicine, dose of a drug and in engineering some environmental conditions (temperature, pressure, . . .) are influenced to the observed variables. The basic problem consist in estimation of distribution of lifetime by such censored dependent data. The aim of paper is considering this problem in the case of right random censoring model in the presence of covariate.

Let's consider the case when the support of covariate $C$ is the interval $[0,1]$ and we describe our results on fixed design points $0 \leq x_{1} \leq x_{2} \leq \ldots \leq x_{n} \leq 1$ at which we consider responses (survival or failure times) $X_{1}, \ldots, X_{n}$ and censoring times $Y_{1}, \ldots, Y_{n}$ of identical objects, which are under study. These responses are independent and nonnegative r.v.-s with conditional distribution function (d.f.) at $x_{i}, F_{x_{i}}(t)=P\left(X_{i} \leq t / C_{i}=x_{i}\right)$. They are subjected to random right censoring, that is for $X_{i}$ there is a censoring variable $Y_{i}$ with conditional d.f. 
$G_{x_{i}}(t)=P\left(Y_{i} \leq t / C_{i}=x_{i}\right)$ and at $n$-th stage of experiment the observed data is

$$
S^{(n)}=\left\{\left(Z_{i}, \delta_{i}, C_{i}\right), 1 \leq i \leq n\right\},
$$

where $Z_{i}=\min \left(X_{i}, Y_{i}\right), \delta_{i}=I\left(X_{i} \leq Y_{i}\right)$ with $I(A)$ denoting the indicator of event $A$. Note that in sample $S^{(n)}$ r.v. $X_{i}$ is observed only when $\delta_{i}=1$. Commonly, in survival analysis to assume independence between the r.v.-s $X_{i}$ and $Y_{i}$ conditional on the covariate $C_{i}$. But, in some practical situations, this assumption does not hold. Therefore, in this article we consider a dependence model in which dependence structure is described through copula function. So let

$$
S_{x}\left(t_{1}, t_{2}\right)=P\left(X_{x}>t_{1}, Y_{x}>t_{2}\right), t_{1}, t_{2} \geq 0
$$

the joint survival function of the response $X_{x}$ and the censoring variable $Y_{x}$ at $x$. Then the marginal survival functions are $S_{x}^{X}(t)=1-F_{x}(t)=S_{x}(t, 0)$ and $S_{x}^{Y}(t)=1-G_{x}(t)=$ $S_{x}(0, t), t \geq 0$. We suppose that the marginal d.f.-s $F_{x}$ and $G_{x}$ are continuous. Then according to the Theorem of Sclar (see, Nelsen (1999)), the joint survival function $S_{x}\left(t_{1}, t_{2}\right)$ can be expressed as

$$
S_{x}\left(t_{1}, t_{2}\right)=C_{x}\left(S_{x}^{X}\left(t_{1}\right), S_{x}^{Y}\left(t_{2}\right)\right), t_{1}, t_{2} \geq 0,
$$

where $C_{x}(u, v)$ is a known copula function depending on $x, S_{x}^{X}$ and $S_{x}^{Y}$ in a general way. It is necessary to note that in the case of no covariates, this idea first was considered by Zeng and Klein (1995) and proposed copula-graphic estimator. Rivest and Wells (2001) investigated copula- graphic estimator and derived a closed form expression for estimator when the joint survival function (1) is modeled as Archimedean copula. The copula-graphic estimator is then shown to be uniformly consistent and asymptotically normal. Note that the copula-graphic estimator is equivalent to the product-limit estimator of Kaplan and Meier (1958) when the survival and censoring times are assumed to be independent. Breakers and Veraverbeke (2005) extend copula- graphic estimator to the fixed design regression case and show that estimator has an asymptotic representation and a Gaussian limit. We consider other estimator of d.f. $F_{x}$ which had a simpler form than copula-graphic estimator and it is also equivalent to the relative-risk power estimator of author Abdushukurov (1998), Abdushukurov (1999) under independent censoring case. We study the large sample properties of estimator proposed and present result of uniform normality with the same limiting Gaussian process as for copulagraphic estimator.

\section{Construction of estimator and asymptotic results}

Assume that at the fixed design value $x \in(0,1), C_{x}$ in (1) is Archimedean copula, i.e.

$$
S_{x}\left(t_{1}, t_{2}\right)=\varphi_{x}^{[-1]}\left(\varphi_{x}\left(S_{x}^{X}\left(t_{1}\right)\right)+\varphi_{x}\left(S_{x}^{Y}\left(t_{2}\right)\right)\right), t_{1}, t_{2} \geq 0,
$$

where, for each $x, \varphi_{x}:[0,1] \rightarrow[0,+\infty]$ is a known continuous, convex, strictly decreasing function with $\varphi_{x}(1)=0 . \varphi_{x}^{[-1]}$ is a pseudo-inverse of $\varphi_{x}$ (see, Nelsen (1999)) and given by

$$
\varphi_{x}^{[-1]}(s)=\left\{\begin{array}{l}
\varphi_{x}^{-1}(s), 0 \leq s \leq \varphi_{x}(0) \\
0, \varphi_{x}(0) \leq s \leq \infty
\end{array}\right.
$$

We assume that copula generator function $\varphi_{x}$ is strict, i.e. $\varphi_{x}(0)=\infty$ and hence $\varphi_{x}^{[-1]}=\varphi_{x}^{-1}$. From (2), it follows that

$$
\begin{gathered}
P\left(Z_{x}>t\right)=1-H_{x}(t)=\overline{H_{x}(t)}=S_{x}{ }^{Z}(t)=S_{x}(t, t)= \\
=\varphi_{x}^{-1}\left(\varphi_{x}\left(S_{x}^{X}(t)\right)+\varphi_{x}\left(S_{x}^{Y}(t)\right)\right), t \geq 0
\end{gathered}
$$


Let $H_{x}^{(1)}(t)=P\left(Z_{x} \leq t, \delta_{x}=1\right)$ be a subdistribution function and $\Lambda_{x}(t)$ is crude hazard function of r.v. $X_{x}$ subjecting to censoring by $Y_{x}$, then (see, Fleming and Harrington (1991)) we have

$$
\Lambda_{x}(d t)=\frac{P\left(X_{x} \in d t, X_{x} \leq Y_{x}\right)}{P\left(X_{x} \geq t, Y_{x} \geq t\right)}=\frac{H_{x}^{(1)}(d t)}{S_{x}^{Z}(t-)} .
$$

From (4) one can obtain following expression of survival function $S_{x}^{X}$ :

$$
\begin{gathered}
S_{x}^{X}(t)=\varphi_{x}^{-1}\left[-\int_{0}^{t} S_{x}^{Z}(u-) \varphi_{x}^{\prime}\left(S_{x}^{Z}(u)\right) d \Lambda_{x}(u)\right]= \\
=\varphi_{x}^{-1}\left[-\int_{0}^{t} \varphi_{x}^{\prime}\left(S_{x}^{Z}(u)\right) d H_{x}^{(1)}(u)\right], t \geq 0,
\end{gathered}
$$

(see, for example, Rivest and Wells (2001); Breakers and Veraverbeke (2005)). In order to constructing the estimator of $S_{x}^{X}$ according to representation (5), we introduce some smoothed estimators of $S_{x}^{Z}, H_{x}^{(1)}$ and regularity conditions for them. Similarly to Breakers and Veraverbeke (2005), we will also use the Gasser-Müller weights

$$
w_{n i}\left(x, h_{n}\right)=\frac{1}{q_{n}\left(x, h_{n}\right)} \int_{x_{i-1}}^{x_{i}} \frac{1}{h_{n}} \pi\left(\frac{x-z}{h_{n}}\right) d z, i=1, \ldots, n,
$$

with

$$
q_{n}\left(x, h_{n}\right)=\int_{0}^{x_{n}} \frac{1}{h_{n}} \pi\left(\frac{x-z}{h_{n}}\right) d z
$$

where $x_{0}=0, \pi$ is a known probability density function (kernel) and $\left\{h_{n}, n \geq 1\right\}$ is a sequence of positive constants, tending to zero as $n \rightarrow \infty$, called bandwidth sequence. Let's introduce the weighted estimators of $H_{x}, S_{x}^{Z}$ and $H_{x}^{(1)}$ respectively as

$$
\begin{aligned}
& H_{x h}(t)=\sum_{i=1}^{n} w_{n i}\left(x, h_{n}\right) I\left(Z_{i} \leq t\right), \\
& S_{x h}^{Z}(t)=1-H_{x h}(t), \\
& H_{x h}^{(1)}(t)=\sum_{i=1}^{n} w_{n i}\left(x, h_{n}\right) I\left(Z_{i} \leq t, \delta_{i}=1\right) .
\end{aligned}
$$

Then pluggin in (5) estimators (7) Abdushukurov and Muradov (2014) proposed following estimator of $S_{x}^{X}$ :

$$
S_{x h}^{X}(t)=1-F_{x h}(t)=\varphi_{x}^{-1}\left[-\int_{0}^{t} \varphi_{x}^{\prime}\left(S_{x}^{Z}(u)\right) d H_{x}^{(1)}(u)\right], t \geq 0 .
$$

Remark that in the case of no covariate, estimator (8) reduces to estimator first obtained by Zeng and Klein (1995), which in case of the independent copula $\varphi(y)=-l o g y$, reduces to a exponential-hazard estimate. Also it is well-known that under independent censoring case Kaplan-Meier's product-limit estimator and exponential-hazard estimators are asymptotical equivalent. Therefore, in Abdushukurov and Muradov (2014) we have showed that estimator (8) and copula-graphic estimator of Breakers and Veraverbeke have the same asymptotic behaviours. 
In this work we propose also the next extended analogue of relative-risk power estimator introduced in Abdushukurov (1998, 1999):

$$
\widehat{S}_{x h}^{Z}(t)=\varphi_{x}^{-1}\left[\varphi_{x}\left(\widehat{S}_{x h}^{Z}(t)\right) \cdot \mu_{x h}(t)\right]=1-\widehat{F}_{x h}(t),
$$

where

$$
\begin{gathered}
\mu_{x h}(t)=\varphi_{x}\left(S_{x h}^{X}(t)\right) / \varphi_{x}\left(\widetilde{S}_{x h}^{Z}(t)\right), \\
\varphi_{x}\left(S_{x h}^{X}(t)\right)=-\int_{0}^{t} \varphi_{x}^{\prime}\left(S_{x h}^{Z}(u)\right) d H_{x h}^{(1)}(u), \\
\varphi_{x}\left(\hat{S}_{x h}^{Z}(t)\right)=-\int_{0}^{t} n\left[\varphi_{x}\left(S_{x h}^{Z}(u)\right)-\varphi_{x}\left(S_{x h}^{Z}(u)-\frac{1}{n}\right)\right] d H_{x h}(u),
\end{gathered}
$$

and

$$
\varphi_{x}\left(\widetilde{S}_{x h}^{Z}(t)\right)=-\int_{0}^{t} \varphi_{x}^{\prime}\left(S_{x h}^{Z}(u)\right) d H_{x h}(u) .
$$

In order to investigate the estimate (9) we introduce some conditions. For the design points $x_{1}, \ldots, x_{n}$, denote

$$
\underline{\Delta_{n}}=\min _{1 \leq i \leq n}\left(x_{i}-x_{i-1}\right), \overline{\Delta_{n}}=\max _{1 \leq i \leq n}\left(x_{i}-x_{i-1}\right) .
$$

For the kernel $\pi$, let

$$
\|\pi\|_{2}^{2}=\int_{-\infty}^{\infty} \pi^{2}(u) d u, m_{\nu}(\pi)=\int_{-\infty}^{\infty} u^{\nu} \pi(u) d u, \nu=1,2,\|\pi\|_{\infty}=\sup _{u \in R} \pi(u) .
$$

Moreover, we use next assumptions on the design and on the kernel function:

(A1) As $n \rightarrow \infty, x_{n} \rightarrow 1, \underline{\Delta_{n}}=O\left(\frac{1}{n}\right), \overline{\Delta_{n}}-\underline{\Delta_{n}}=o\left(\frac{1}{n}\right)$.

(A2) $\pi$ is a probability density function with compact support $[-M, M]$ for some $M>0$, with $m_{1}(\pi)=0$ and $\left|\pi(u)-\pi\left(u^{\prime}\right)\right| \leq C(\pi)\left|u-u^{\prime}\right|$, where $C(\pi)$ is some constant.

Let $T_{H_{x}}=\inf \left\{t \geq 0: H_{x}(t)=1\right\}$. Then $T_{H_{x}}=\min \left(T_{F_{x}}, T_{G_{x}}\right)$. For our results we need some smoothnees conditions on functions $H_{x}(t)$ and $H_{x}^{(1)}(t)$. We formulate them for a general (sub)distribution function $N_{x}(t), 0 \leq x \leq 1, t \in R$ and for a fixed $T>0$.

(A3) $\frac{\partial}{\partial x} N_{x}(t)=\dot{N}_{x}(t)$ exists and is continuous in $(x, t) \in[0,1] \times[0, T]$.

(A4) $\frac{\partial}{\partial x} N_{x}(t)=N_{x}^{\prime}(t)$ exists and is continuous in $(x, t) \in[0,1] \times[0, T]$.

(A5) $\frac{\partial^{2}}{\partial x^{2}} N_{x}(t)=\ddot{N}_{x}(t)$ exists and is continuous in $(x, t) \in[0,1] \times[0, T]$.

(A6) $\frac{\partial^{2}}{\partial t^{2}} N_{x}(t)=N_{x}^{\prime \prime}(t)$ exists and is continuous in $(x, t) \in[0,1] \times[0, T]$.

(A7) $\frac{\partial^{2}}{\partial x \partial t} N_{x}(t)=\dot{N}_{x}^{\prime}(t)$ exists and is continuous in $(x, t) \in[0,1] \times[0, T]$.

(A8) $\frac{\partial \varphi_{x}(u)}{\partial u}=\varphi_{x}^{\prime}(u)$ and $\frac{\partial^{2} \varphi_{x}(u)}{\partial u^{2}}=\varphi_{x}^{\prime \prime}(u)$ are Lipschitz in the $x$-direction with a bounded Lipschitz constant and $\frac{\partial^{3} \varphi_{x}(u)}{\partial u^{3}}=\varphi_{x}^{\prime \prime \prime}(u)$ exists and is continuous in $(x, u) \in[0,1] \times(0,1]$. 
We derive an almost sure representation result with rate.

Theorem 1. Assume (A1), (A2), $H_{x}(t)$ and $H_{x}^{(1)}(t)$ satisfy (A5)-(A7) in $[0, T]$ with $T<T_{H_{x}}$, $\varphi_{x}$ satisfies (A8) and $h_{n} \rightarrow 0, \frac{\operatorname{logn}}{n h_{n}} \rightarrow 0, \frac{n h_{n}^{5}}{\operatorname{logn}}=O(1)$. Then, as $n \rightarrow \infty$,

$$
\widehat{F}_{x h}(t)-F_{x}(t)=\sum_{i=1}^{n} w_{n i}\left(x, h_{n}\right) \Psi_{t x}\left(Z_{i}, \delta_{i}\right)+r_{n}(t)
$$

where

$$
\begin{gathered}
\Psi_{t x}\left(Z_{i}, \delta_{i}\right)=\frac{-1}{\varphi_{x}^{\prime}\left(S_{x}^{X}(t)\right)}\left[\int_{0}^{t} \varphi_{x}^{\prime \prime}\left(S_{x}^{Z}(u)\right)\left(I\left(Z_{i} \leq u\right)-H_{x}(u)\right) d H_{x}^{(1)}(u)-\right. \\
-\varphi_{x}^{\prime}\left(S_{x}^{Z}(t)\right)\left(I\left(Z_{i} \leq t, \delta_{i}=1\right)-H_{x}^{(1)}(t)\right)- \\
\left.-\int_{0}^{t} \varphi_{x}^{\prime \prime}\left(S_{x}^{Z}(u)\right)\left(I\left(Z_{i} \leq u, \delta_{i}=1\right)-H_{x}^{(1)}(u)\right) d H_{x}(u)\right]
\end{gathered}
$$

and

$$
\sup _{0 \leq t \leq T}\left|r_{n}(t)\right| \stackrel{\text { a.s. }}{=} O\left(\left(\frac{\log n}{n h_{n}}\right)^{3 / 4}\right) \text {. }
$$

The weak convergence of the empirical process $\left(n h_{n}\right)^{1 / 2}\left\{\widehat{F}_{x h}(\cdot)-F_{x}(\cdot)\right\}$ in the space $l^{\infty}[0, T]$ of uniformly bounded functions on $[0, T]$, endowed with the uniform topology is the content of the next theorem.

Theorem 2. Assume (A1), (A2), $H_{x}(t)$ and $H_{x}^{(1)}(t)$ satisfy (A5)-(A7) in $[0, T]$ with $T<T_{H_{x}}$, and that $\varphi_{x}$ satisfies (A8).

(I) If $n h_{n}^{5} \rightarrow 0$ and $\frac{(\log n)^{3}}{n h_{n}} \rightarrow 0$, then, as $n \rightarrow \infty$,

$$
\left(n h_{n}\right)^{1 / 2}\left\{\widehat{F}_{x h}(\cdot)-F_{x}(\cdot)\right\} \Rightarrow \boldsymbol{W}_{x}(\cdot) \text { in } l^{\infty}[0, T] .
$$

(II)If $h_{n}=C n^{-1 / 5}$ for some $C>0$, then, as $n \rightarrow \infty$,

$$
\left(n h_{n}\right)^{1 / 2}\left\{\widehat{F}_{x h}(\cdot)-F_{x}(\cdot)\right\} \Rightarrow \boldsymbol{W}_{x}^{*}(\cdot) \text { in } l^{\infty}[0, T],
$$

where $\boldsymbol{W}_{x}(\cdot)$ and $\boldsymbol{W}_{x}^{*}(\cdot)$ are Gaussian processes with means

$$
E \boldsymbol{W}_{x}(t)=0, E \boldsymbol{W}_{x}^{*}(t)=a_{x}(t),
$$

and same covariance

$$
\operatorname{Cov}\left(\boldsymbol{W}_{x}(t), \boldsymbol{W}_{x}^{*}(s)\right)=\operatorname{Cov}\left(\boldsymbol{W}_{x}^{*}(t), \boldsymbol{W}_{x}^{*}(s)\right)=\Gamma_{x}(t, s),
$$

with

$$
a_{x}(t)=\frac{-C^{5 / 2} m_{2}(\pi)}{2 \varphi_{x}^{\prime}\left(S_{x}^{X}(t)\right)} \int_{0}^{t}\left[\varphi_{x}^{\prime \prime}\left(S_{x}^{Z}(u)\right) \ddot{H} x(u) d H_{x}^{(1)}(u)-\varphi_{x}^{\prime}\left(S_{x}^{Z}(u)\right) d H_{x}^{(\ddot{1})}(u)\right]
$$

and

$$
\begin{gathered}
\Gamma_{x}(t, s)=\frac{\|\pi\|_{2}^{2}}{\varphi_{x}^{\prime}\left(S_{x}^{X}(t)\right) \varphi_{x}^{\prime}\left(S_{x}^{X}(s)\right)}\left\{\int_{0}^{\min (t, s)}\left(\varphi_{x}^{\prime}\left(S_{x}^{Z}(z)\right)\right)^{2} d H_{x}^{(1)}(z)+\right. \\
+\int_{0}^{\min (t, s)}\left[\varphi_{x}^{\prime \prime}\left(S_{x}^{Z}(w)\right) S_{x}^{Z}(w)+\varphi_{x}^{\prime}\left(S_{x}^{Z}(w)\right)\right] \int_{0}^{w} \varphi_{x}^{\prime \prime}\left(S_{x}^{Z}(y)\right) d H_{x}^{(1)}(y) d H_{x}^{(1)}(w)+
\end{gathered}
$$




$$
\begin{gathered}
+\int_{0}^{\min (t, s)} \varphi_{x}^{\prime \prime}\left(S_{x}^{Z}(w)\right) \int_{w}^{\max (t, s)}\left(\varphi_{x}^{\prime \prime}\left(S_{x}^{Z}(y)\right) S_{x}^{Z}(y)+\varphi_{x}^{\prime}\left(S_{x}^{Z}(y)\right)\right) d H_{x}^{(1)}(y) d H_{x}^{(1)}(w)- \\
-\int_{0}^{t}\left[\varphi_{x}^{\prime \prime}\left(S_{x}^{Z}(y)\right) S_{x}^{Z}(y)+\varphi_{x}^{\prime}\left(S_{x}^{Z}(y)\right)\right] d H_{x}^{(1)}(y) . \\
\left.\cdot \int_{0}^{s}\left[\varphi_{x}^{\prime \prime}\left(S_{x}^{Z}(w)\right) S_{x}^{Z}(w)+\varphi_{x}^{\prime}\left(S_{x}^{Z}(w)\right)\right] d H_{x}^{(1)}(w)\right\} .
\end{gathered}
$$

It is clear that for existence of right hand side of representation (5) we must require the conditions (A4) for functions $H_{x}(t)$ and $H_{x}^{(1)}(t)$ in $[0,1] \times[0, T]$ with $T<T_{H_{x}}$ and existence of $\varphi_{x}^{\prime}(u)$ on $[0,1] \times(0,1]$.

\section{Proofs of Theorems 1 and 2}

In paper Abdushukurov and Muradov (2014) authors have proved analogues of Theorems 1 and 2 for estimator (8). Therefore, it is sufficient for us to prove asymptotic equivalence of estimators (8) and (9). This is the content of next lemma.

Lemma. Assume (A1), (A2), $H_{x}(t)$ and $H_{x}^{(1)}(t)$ satisfy (A5)-(A7) in $[0, T]$ with $T<T_{x}$, $\varphi_{x}$ satisfies (A8). Then, as $n \rightarrow \infty$

$$
\sup _{0 \leq t \leq T}\left|\hat{S}_{x h}^{X}(t)-S_{x h}^{X}(t)\right| \stackrel{a . s .}{=} O\left(\frac{1}{n}\right) .
$$

Proof. For all $(x ; t) \in[0,1] \times(0, T]$ we have

$$
\begin{gathered}
\hat{S}_{x h}^{X}(t)-S_{x h}^{X}(t)=\varphi_{x}^{-1}\left[\varphi_{x}\left(\hat{S}_{x h}^{Z}(t)\right) \mu_{x h}(t)\right]- \\
-\varphi_{x}^{-1}\left[-\int_{0}^{t} \varphi_{x}^{\prime}\left(S_{x h}^{Z}(u)\right) d H_{x h}^{(1)}(u)\right]= \\
=-\frac{1}{\varphi_{x}^{\prime}\left(\zeta_{x h}(t)\right)}\left[\varphi_{x}\left(\hat{S}_{x h}^{Z}(t) \mu_{x h}(t)-\varphi_{x}\left(S_{x h}^{X}(t)\right)\right)\right]= \\
=\frac{\mu_{x h}(t)}{\varphi_{x}^{\prime}\left(\varsigma_{x h}(t)\right)}\left[\varphi_{x}\left(\hat{S}_{x h}^{Z}(t)\right)-\varphi_{x}\left(\tilde{S}_{x h}^{Z}(t)\right)\right],
\end{gathered}
$$

where $\varsigma_{x h}(t) \in\left(\min \left\{\varphi_{x}\left(\hat{S}_{x h}^{Z}(t)\right) \mu_{x h}(t), S_{x h}^{X}(t)\right\}, \max \left\{\varphi_{x}\left(\hat{S}_{x h}^{Z}(t)\right) \mu_{x h}(t), S_{x h}^{X}(t)\right\}\right)$. It is not difficult to see, that for all $(x, t) \in[0,1] \times(0, T]$ and $n \geq 1$,

$$
0 \leq \mu_{x h}(t) \leq 1
$$

hence

$$
\begin{gathered}
\sup _{0 \leq t \leq T}\left|\hat{S}_{x h}^{X}(t)-S_{x h}^{X}(t)\right| \leq \sup _{0 \leq t \leq T}\left|\varphi_{x}^{\prime}\left(\varsigma_{x h}(t)\right)\right|^{-1} . \\
\cdot \sup _{0 \leq t \leq T}\left|\varphi_{x}\left(\hat{S}_{x h}^{Z}(t)\right)-\varphi_{x}\left(\widetilde{S}_{x h}^{Z}(t)\right)\right|
\end{gathered}
$$

But

$$
\begin{gathered}
\sup _{0 \leq t \leq T}\left|\varphi_{x}\left(\hat{S}_{x h}^{Z}(t)\right)-\varphi_{x}\left(\widetilde{S}_{x h}^{Z}(t)\right)\right| \leq \\
\sup _{0 \leq t \leq T} \int_{0}^{T}\left|n\left[\varphi_{x}\left(S_{x h}^{Z}(u)\right)-\varphi_{x}\left(S_{x h}^{Z}(u)-\frac{1}{n}\right)-\varphi_{x}^{\prime}\left(S_{x h}^{Z}(u)\right)\right]\right| d H_{x h}(u) \leq
\end{gathered}
$$




$$
\leq \frac{1}{2 n} \sup _{0 \leq t \leq T}\left|\varphi_{x}^{\prime}\left(\theta_{x h}(t)\right)\right| \stackrel{\text { a.s. }}{=} O\left(\frac{1}{n}\right)
$$

where

$$
\theta_{x h}(t) \in\left(\min \left\{S_{x h}^{Z}(t), S_{x h}^{Z}(t)-\frac{1}{n}\right\}, \max \left\{S_{x h}^{Z}(t), S_{x h}^{Z}(t)-\frac{1}{n}\right\}\right) .
$$

Now from (11) and (12) follows (10). Lemma is proved.

So far as for all $(x, t) \in[0,1] \times(0, T]$

$$
\stackrel{\wedge}{F}_{x h}(t)-F_{x}(t)=F_{x h}(t)-F_{x}(t)+q_{n}(t),
$$

where $q_{n}(t)=\stackrel{\wedge}{F}_{x h}(t)-F_{x h}(t)$ and by Lemma $\sup _{0 \leq t \leq T}\left|q_{n}(t)\right| \stackrel{a . s .}{=} O\left(\frac{1}{n}\right)$. Moreover, from Theorem 1.1 in Abdushukurov and Muradov (2014),

$$
F_{x h}(t)-F_{x}(t)=\sum_{i=1}^{n} w_{n i}\left(x, h_{n}\right) \Psi_{t x}\left(Z_{i}, \delta_{i}\right)+r_{n}(t),
$$

where summands of right hand side are defined as in Theorem1. Consequently, Theorem 1 follows from relations (12)-(14).

It is necessary to note that almost sure representation of Theorem 1 plays a key role on investigating of estimator (9) and, in particular, it provides a basic tool for obtaining of weak convergence results of Theorem 2. But the main summands $\Psi_{t x}$ of representation is the same as in the case of copula-graphic estimator from Breakers and Veraverbeke (2005) (see, (14)). Then the proof of Theorem 2 one can accomponing by line of proof of Theorem 1.2 from Breakers and Veraverbeke (2005) and hence it omitted. Thus, the copula-graphic estimator from Breakers and Veraverbeke (2005) with estimators (8) and (9) are asymptotic equivalent.

\section{References}

Abdushukurov AA (1998). "Nonparametric Estimation of Distribution Function Based on Relative Risk Function." Communications in Statistics: Theory and Methods, 27(8), 19912012.

Abdushukurov AA (1999). "On Nonparametric Estimation of Reliability Indices by Censored Samples." Theory of Probability and Its Applications, 43(1), 3-11.

Abdushukurov AA, Muradov RS (2014). "On Estimation of Conditional Distribution Function under Dependent Random Right Censored Data." Journal of Siberian Federal University. Mathematics \& Physics, 7(4), 409-416.

Breakers R, Veraverbeke N (2005). "A Copula-graphic Estimator for the Conditional Survival Function under Dependent Censoring." The Canadian Journal of Statistics, 33(3), 429-447.

Fleming TR, Harrington DP (1991). Counting Processes and Survival Analysis. Wiley, New York.

Kaplan EL, Meier P (1958). "Nonparametric Estimation from Incomplete Observations." Journal of American Statistical Association, 53, 457-481.

Nelsen RB (1999). An Introduction to Copulas. Springer, New York.

Rivest LP, Wells MT (2001). "A Martingall Approach to the Copula-graphic Estimator for the Survival Function under Dependent Censoring." Journal of Multivariate Analysis, 79, 138-155. 
Zeng M, Klein JP (1995). "Estimates of Marginal Survival for Dependent Competing Risks Based on an Assumed Copula." Biometrika, 82, 127-138.

\author{
Affiliation: \\ Abdushukurov Abdurahim Ahmedovich \\ Department Applied Mathematics and Informatics \\ Tashkent Branch of Moscow State University named after M.V. Lomonosov \\ Amir Temur Avenue,22 \\ 100060 Tashkent, Uzbekistan \\ E-mail: a_abdushukurov@rambler.ru
}

\section{Austrian Journal of Statistics}

published by the Austrian Society of Statistics

Volume 49

April 2020 http://www.ajs.or.at/

http://www.osg.or.at/

Submitted: 2019-12-15

Accepted: 2020-03-03 